\title{
Clustering Phenomena in Coupled Chaotic Circuits with Different Coupling Strength
}

\author{
Yoko Uwate ${ }^{\dagger}$, Thomas Ott ${ }^{\ddagger}$ and Yoshifumi Nishio ${ }^{\dagger}$ \\ $\dagger$ Dept. of Electrical and Electronic Engineering,Tokushima University \\ 2-1 Minami-Josanjima, Tokushima, Japan \\ Email: \{uwate, nishio\}@ee.tokushima-u.ac.jp \\ $\ddagger$ Institute of Applied Simulation, Zurich University of Applied Sciences, \\ Einsiedlerstrasse 31a, 8820 Waedenswil, Switzerland \\ Email: thomas.ott@zhaw.ch
}

\begin{abstract}
In this study, we investigate synchronization phenomena in coupled chaotic circuits with different coupling strength when chaotic circuits are arranged irregularly in one dimensional array. Three groups are composed in this circuit system and each group includes some chaotic circuits. The coupling strength corresponds to the distance between the chaotic circuits. We observe several types of clustering patterns when the coupling strength is fixed to appropriate value.
\end{abstract}

\section{INTRODUCTION}

Synchronization is one of the basic phenomena and it can be observed everywhere in our living life [1]-[5]. Therefore, studies on synchronization phenomena have investigated in the various fields. In particular, we are interested in synchronization phenomena observed in electronic circuits. We consider that there are many real physical phenomena exhibiting by coupled chaotic circuits, and it is important to investigate synchronization phenomena observed from coupled chaotic circuits for future engineering applications.

Recently, we have investigated synchronization phenomena observed from complex networks of the coupled chaotic circuits [6], [7]. The chaotic circuits are placed on 2-dimensional space and the coupled parameters depend on the distance between the circuits. We have observed interesting clustering phenomena from this proposed system by computer simulations and circuit experimental results. In order to understand the obtained results in detail, we need to consider more simple circuit system.

In our previous study, we have investigated synchronization phenomena of an array of the coupled chaotic circuits [8]. In this circuit system, the chaotic circuits are arranged in two groups. By using computer simulations, we have confirmed chaos synchronization when the number of the chaotic circuits of two groups is same. While, the breakdown of chaos synchronization is occurred by changing from the symmetric arrangement to the asymmetric system which the number of the chaotic circuits of two groups is different. We consider that it is interesting to change the synchronization states depending on the arrangement method of the circuits.

In this study, we expand the circuit system to more complex system. Three groups are composed in this circuit system and each group includes some chaotic circuits. We focus on the phase difference between intergroup and intragroup circuits. By using computer simulations, we observe several types of synchronization states when the coupling strength is fixed to appropriate value. Furthermore, we discuss the relationship between the obtained synchronization states and clustering phenomena.

\section{CiRCUit MOdEL}

Figure 1 shows the chaotic circuit called Nishio-Inaba circuit, investigated in [9]-[11]. The circuit consists of a negative resistance, a nonlinear resistance consisting of two diodes, a capacitor and two inductors.

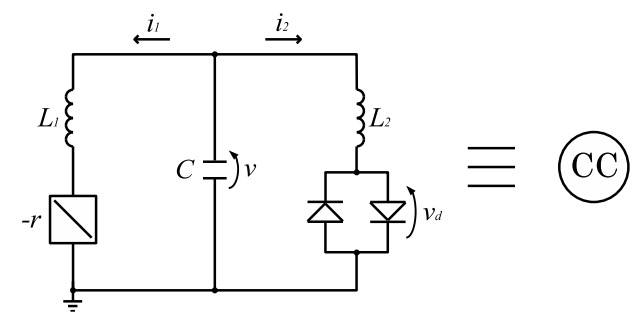

Fig. 1. Nishio-Inaba chaotic circuit.

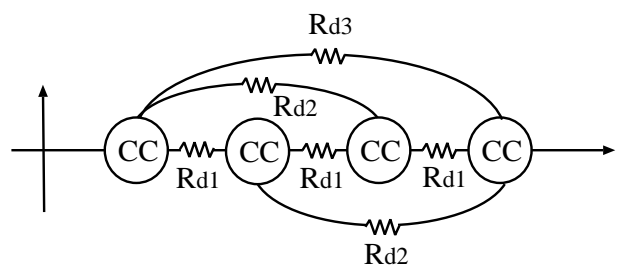

Fig. 2. Arrangement of chaotic circuits with globally coupling as one dimensional array.

In this study, we consider that the chaotic circuits are arranged in one dimensional array as shown in Fig. 2. All chaotic circuits are connected to each other by resistors. Furthermore, the value of the coupling strength depends on the distance btween the circuits. 
By using the variables and the parameters as follows;

$$
\begin{gathered}
i_{1}=\sqrt{\frac{C}{L_{1}}} V x ; i_{2}=\frac{\sqrt{L_{1} C}}{L_{2}} V y ; v=V z ; \\
r \sqrt{\frac{C}{L_{1}}}=\alpha ; \frac{L_{1}}{L_{2}}=\beta ; r_{d} \frac{\sqrt{L_{1} C}}{L_{2}}=\delta ; \\
t=\sqrt{L_{1} C} \tau ; " . "=\frac{d}{d \tau}
\end{gathered}
$$

We obtain the normalized circuit equations.

$$
\begin{aligned}
\frac{d x_{i}}{d \tau} & =\alpha x_{i}+z_{i} \\
\frac{d y_{i}}{d \tau} & =z_{i}+f(y) \\
\frac{d z_{i}}{d \tau} & =-x_{i}-\beta y_{i}-\sum_{j=1}^{N} \gamma_{i j}\left(z_{i}-z_{j}\right) \\
(i, j & =1,2, \cdots, N)
\end{aligned}
$$

where $f(y)$ is described as follows:

$$
f(y)=\frac{\delta}{2}\left(\left|y+\frac{1}{\delta}\right|-\left|y-\frac{1}{\delta}\right|\right) .
$$

In this equation, $i$ in the equation represents the circuit itself, and $j$ indicates the coupling with other circuits. The parameter $\gamma_{i j}$ represents the coupling strength between the circuits. The value of $\gamma_{i j}$ reflects the distance between the circuits in an inverse way, described by the following equation:

$$
\gamma_{i j}=\frac{g}{\left(d_{i j}\right)^{2}} \text {. }
$$

$d_{i j}$ denotes the Euclidean distance between the $i-t h$ circuit and the $j-t h$ circuit. The parameter $g$ is a weighting or scaling parameter that determines the coupling strengths.

\section{BASIC SYNCHRONIZATION PHENOMENA}

First, we explain the basic synchronization phenomena when the chaotic circuits are coupled at irregular intervals. The two types arrangements of chaotic circuits (symmetric and asymmetric models) are considered as shown in Fig 3. In these circuit models, two groups are arranged in one dimensional space. The nearest distance between the chaotic circuits within the group is set to $d_{i n}=0.01$. The distance between the intergroup circuits is set to $d_{\text {out }}=0.1$. In this simulation, we set the scaling parameter to $g=1.0 \times 10^{-5}$ and the coupling strength between two circuits is determined by Eq. (3).

Figure 4 shows the simulation results of the symmetric system (Fig. 3 (a)). The chaotic circuits within the group are synchronized with in-phase state as shown in Fig. 4 (a). Also, the in-phase synchronization is observed from the intergroup circuits (see Fig. 4 (b)). Similarly, Fig. 5 shows the results of the phase differences between the chaotic circuits of the asymmetric system (Fig. 3 (b)). In this case, the asynchronization can be observed from the intergroup circuits (see Fig. 5 (b)).

From these results, all chaotic circuits are synchronized when the chaotic circuits are coupled by symmetry. However, the case of that the chaotic circuits are coupled by asymmetry, asynchronous phenomena can be observed from the adjacent chaotic circuits of the intergroup circuits. We consider that it is interesting to change the synchronization states depending on the arrangement method of the chaotic circuits.

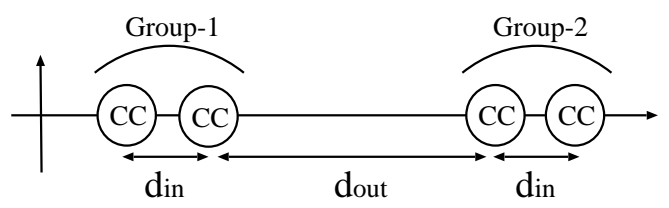

(a) Symmetric system (2-2 network).

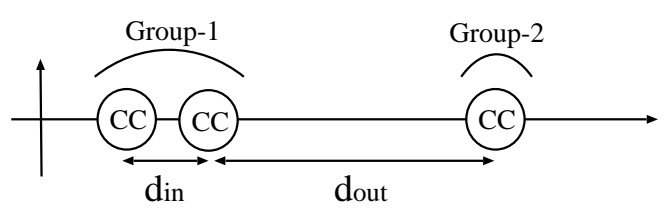

(b) Asymmetric system (2-1 network).

Fig. 3. Arrangement of chaotic circuits with two groups.

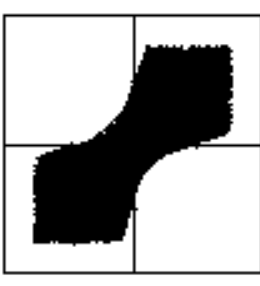

(a) Group1-Group1.

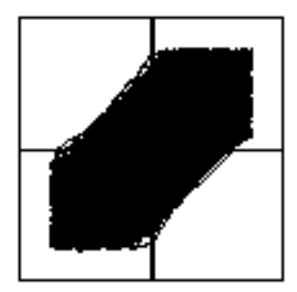

(b) Group1-Group2.
Fig. 4. Phase difference of intragroup and intergroup circuits for symmetric arrangement. ((a): $9.44^{\circ},\left(\right.$ b): $\left.28.19^{\circ}\right)$

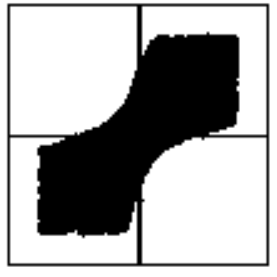

(a) Group1-Group1.

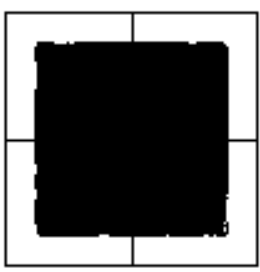

(b) Group1-Group2.
Fig. 5. Phase difference of intragroup and intergroup circuits for asymmetric arrangement. ((a): $9.54^{\circ},\left(\right.$ b): $\left.90.00^{\circ}\right)$

\section{SYNCHRONIZATION PHENOMENA}

In this section, several types of the circuit arrangement are considered as follows.

\section{A. Synchronization phenomena I}

Here, we investigate synchronization phenomena when the chaotic circuits are arranged in three groups. The two types arrangements of chaotic circuits (symmetric and asymmetric models) are considered as shown in Fig 6. In this circuit 


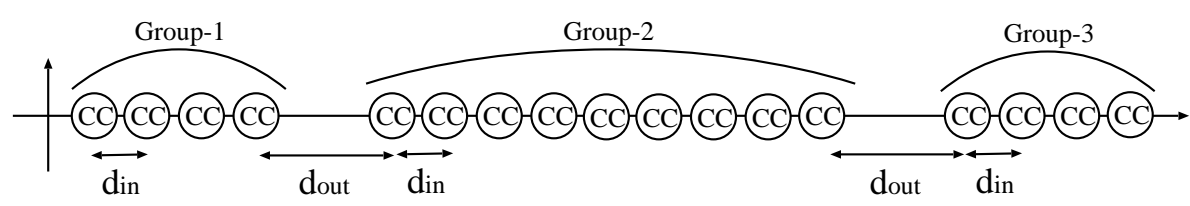

(a) Symmetric system (4-9-4 network).

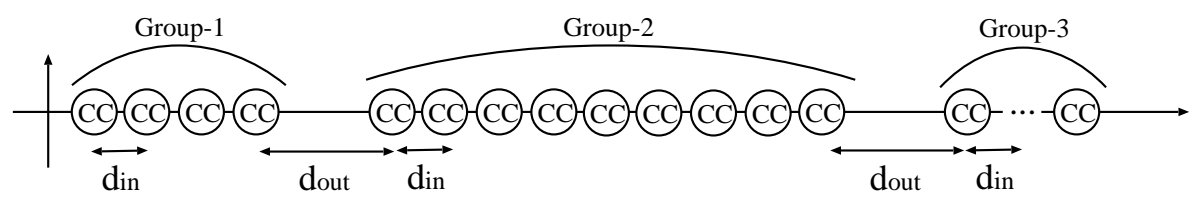

(b) Asymmetric system (4-9-x network).

Fig. 6. Arrangement of chaotic circuit with three groups.

system, the distance parameters are fixed with $d_{i n}=0.01$ and $d_{\text {out }}=0.1$, and the coupling strength is set to $g=1.0 \times 10^{-5}$. For the asymmetric model, the number of the chaotic circuits in group 3 is changed from 1 to 3 .

Figures 7-10 show the simulation results of the phase difference of the intergroup circuits. In the case of symmetric 4-9-4 network, all intergroup circuits are synchronized with in-phase state as shown in Fig. 7. Figure 8 shows the phase differences of asymmetric 4-9-3 network. The chaotic circuits between group1 and group3 are synchronized with in-phase. While, the other intergroup circuits are not synchronized. By decreasing the number of chaotic circuits in group3, the different type of synchronization is observed. In the case of 4-9-2 and 4-91 networks, the chaotic circuits between group1 and group2 are synchronized with in-phase state. Asynchronization can be obtained from the other intergroup circuits (see Figs. 9, 10).

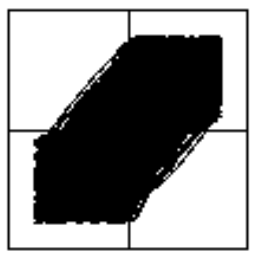

(a) Group1-Group2.

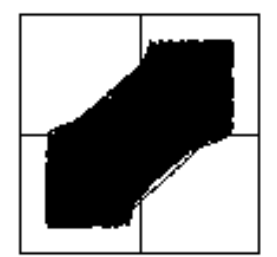

(b) Group2-Group3.

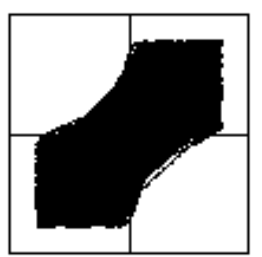

(c) Group3-Group1.
Fig. 7. Phase difference of intergroup circuits for 4-9-4 network. (a): $23.66^{\circ},(\mathrm{b}): 24.48^{\circ}$, (c): $\left.14.26^{\circ}\right)$

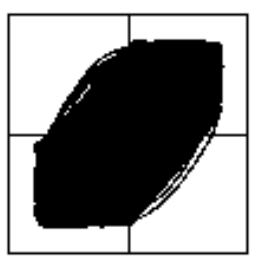

(a) Group1-Group2.

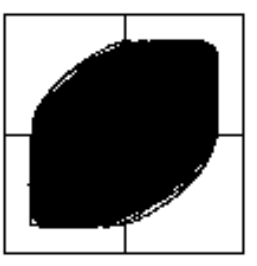

(b) Group2-Group3.

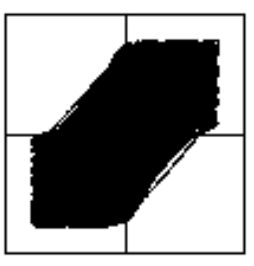

(c) Group3-Group1.
Fig. 8. Phase difference of intergroup circuits for 4-9-3 network ((a): $\left.40.13^{\circ},(\mathrm{b}): 58.39^{\circ},(\mathrm{c}): 17.57^{\circ}\right)$

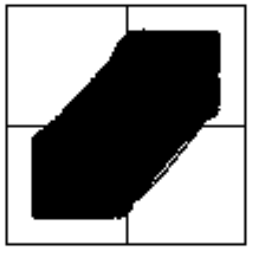

(a) Group1-Group2.

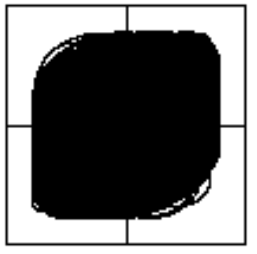

(b) Group2-Group3.

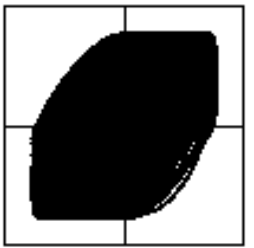

(c) Group3-Group1.
Fig. 9. Phase difference of intergroup circuits for 4-9-2 network ((a): $27.66^{\circ}$, (b): $90.00^{\circ}$, (c): $62.77^{\circ}$ )

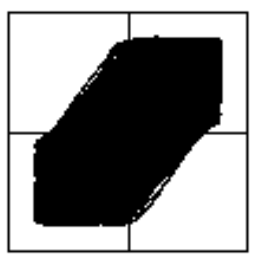

(a) Group1-Group2.

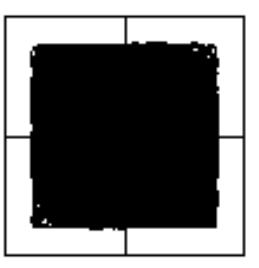

(b) Group2-Group3.

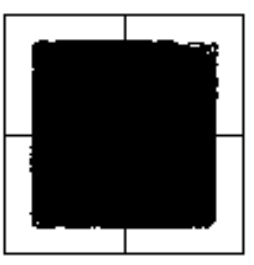

(c) Group3-Group1.
Fig. 10. Phase difference of intergroup circuits for 4-9-1 network. ((a): $\left.29.16^{\circ},(\mathrm{b}): 90.00^{\circ},(\mathrm{c}): 90.00^{\circ}\right)$

Next, we consider the relationship between the obtained synchronization states and clustering phenomena. We define the synchronized circuits as one cluster. Table I summarizes the clustering phenomena for all types of the networks. We observe one cluster in the symmetric system. In the case of the asymmetric system, two clusters are observed. From these results, we confirm that several types of clustering phenomena can be observed from the asymmetric circuit systems.

\section{B. Synchronization phenomena II}

Next, we investigate the synchronization state of symmetric 4-9-4 network when the coupling strength is changed. Figure 11 shows the simulation results of the phase differences. When the coupling strength is small value, we confirm the asynchrous state for whole circuit system. First, the phase difference of the intergroup circuits decreases around $g=$ 
TABLE I

SUMMARY OF CLUSTERING PHENOMENA.

\begin{tabular}{c|l|l}
\hline Network & Clustering number & Clustering type \\
\hline \hline $4-9-4$ & 1 cluster & (groups 1-2-3) \\
\hline $4-9-3$ & 2 clusters & (groups 1-3), (group 2) \\
$4-9-2$ & 2 clusters & (groups 1-2), (group 3) \\
$4-9-2$ & 2 clusters & (groups 1-2), (group 3) \\
\hline
\end{tabular}

$1.0 \times 10^{-6}$. At this region, the synchronization of the chaotic circuits within the group is occurred. Next, the phase difference between the group1 and the group3 shows in-phase state. However, the chaotic circuits between the other groups are not synchronized (see Fig. 12). Finally, we confirm fully synchronization state around $g=1.0 \times 10^{-5}$.

Table II summarizes the relationship between the obtained synchronization state and the clustering phenomena. We confirm that the several types of clustering phenomena can be obtained by setting the coupling strength.

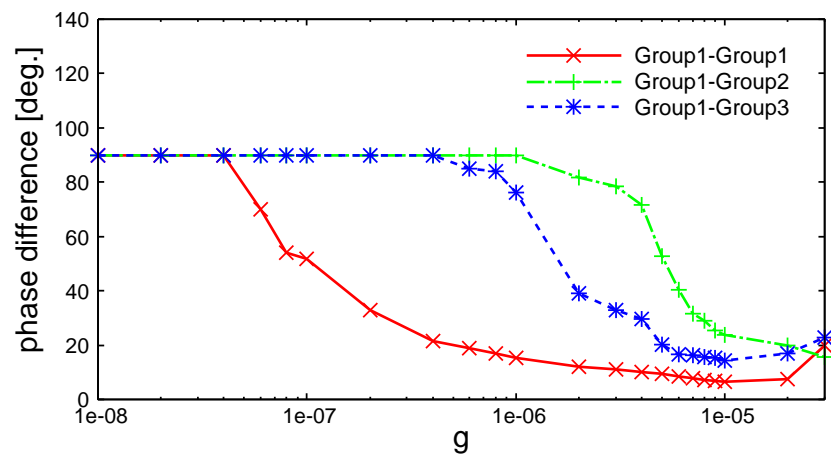

Fig. 11. Average of phase difference with coupling strength.

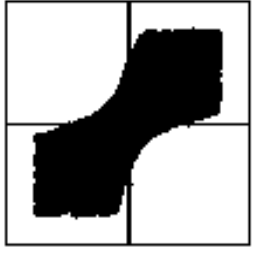

(a) Group1-Group1.

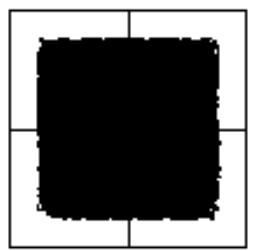

(d) Group1-Group2.

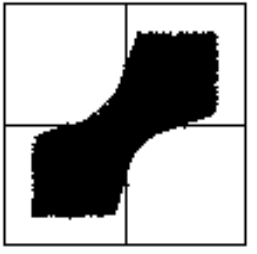

(b) Group2-Group2.

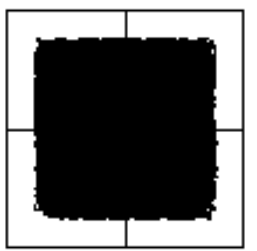

(e) Group2-Group3.

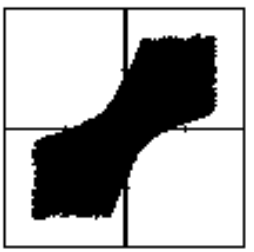

(c) Group3-Group3.

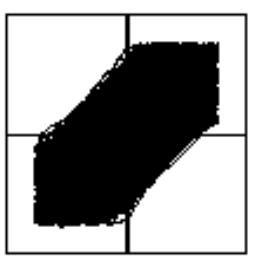

(f) Group3-Group1
Fig. 12. Synchronization state of two clusters. ((a): $10.09^{\circ}$, (b): $9.37^{\circ}$, (c): $\left.9.96^{\circ},(\mathrm{d}): 90.00^{\circ},(\mathrm{e}): 90.00^{\circ},(\mathrm{f}): 29.68^{\circ}\right)$
TABLE II: SUMMARY OF SYNCHRONIZATION STATE.

\begin{tabular}{c||l|ccc}
\hline $\mathrm{g}$ & Synchronization States & \multicolumn{2}{|c}{ Clustering type } \\
\hline \hline $1 \mathrm{e}-8: 0.6 \mathrm{e}-7$ & Fully asynchronization & 4 & 9 & 4 \\
\hline $0.8 \mathrm{e}-7: 0.3 \mathrm{e}-6$ & Synchronization of chaotic circuits in same group & 4 & 9 & 4 \\
\hline $0.4 \mathrm{e}-6: 0.9 \mathrm{e}-6$ & Synchronization between Group1 and Group3 & 4 & 9 & 4 \\
\hline $1 \mathrm{e}-5: 0.3 \mathrm{e}-4$ & Fully synchronization & 4 & 9 & 4 \\
\hline
\end{tabular}

\section{Conclusion}

In this study, we have investigated synchronization phenomena in coupled chaotic circuits with different coupling strength when chaotic circuits are arranged irregularly in one dimensional array. Three groups are composed in this circuit system and each group includes some chaotic circuits. The coupling strength corresponds to the distance between the chaotic circuits. We have observed several types of synchronization states when the coupling strength is fixed to appropriate value.

\section{ACKNOWLEDGMENT}

This work was partly supported by JSPS Grant-in-Aid for Young Scientists 23700269

\section{REFERENCES}

[1] L.L. Bonilla, C.J. Perez Vicente and R. Spigler, "Time-periodic phases in populations of nonlinearly coupled oscillators with bimodal frequency distributions," Physica D: Nonlinear Phenomena, vol. 113, no. 1, pp. 79-97, Feb. 1998.

[2] J.A. Sherratt, "Invading wave fronts and their oscillatory wakes are linked by a modulated traveling phase resetting wave," Physica D: Nonlinear Phenomena, vol. 117, no. 1-4, pp. 145166, Jul. 1998.

[3] G. Abramson, V.M. Kenkre and A.R. Bishop, "Analytic solutions for nonlinear waves in coupled reacting systems," Physica A: Statistical Mechanics and its Applications, vol. 305, no. 3-4, pp. 427-436, Mar. 2002.

[4] I. Belykh, M. Hasler, M. Lauret and H. Nijmeijer, "Synchronization and graph topology," Int. J. Bifurcation and Chaos, vol. 15, no. 11, pp. 3423-3433, Nov. 2005.

[5] C.M. Gray, "Synchronous oscillations in neural systems: mechanisms and functions," J. Computational Neuroscience, vol. 1, pp. 11-38, 1994.

[6] Y. Takamaru, H. Kataoka, Y. Uwate and Y. Nishio, "Clustering phenomena in complex networks of chaotic circuits", Proc. of ISCAS'12, pp. 914-917, Mar. 2012.

[7] Y. Takamaru, Y. Uwate, T. Ott and Y. Nishio, "Clustering phenomena of coupled chaotic circuits for large scale networks", Proc. of NDES'12, pp. 70-73, Jul. 2012.

[8] K. Okamoto, Y. Takamaru, Y Uwate and Y. Nishio, "Synchronization of an Array of Chaotic Circuits Coupled by Different Coupling Strength," Proc. of NCSP'13., 1pp. 681-684, Mar. 2013.

[9] Y. Nishio, N. Inaba, S. Mori and T. Saito, "Rigorous analyses of windows in a symmetric circuit," IEEE Transactions on Circuits and Systems, vol. 37, no. 4, pp. 473-487, Apr. 1990.

[10] C. Bonatto and J. A. C. Gallas, "Periodicity hub and nested spirals in the phase diagram of a simple resistive circuit," Phys. Rev. Lett., 101, 054101, Aug. 2008.

[11] R. Stoop, P. Benner and Y Uwate, "Real-world existence and origins of the spiral organization of shrimp-shaped domains," Phys. Rev. Lett., 105, 074102, Aug. 2010. 